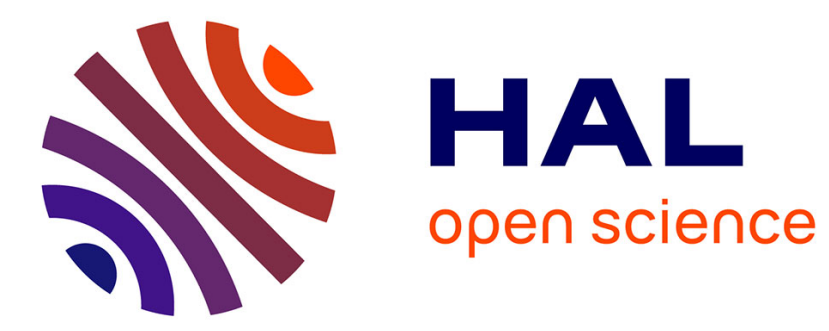

\title{
Friction-induced self-organization of a one-dimensional array of particles
}

Farhang Radjai, Stéphane Roux

\section{To cite this version:}

Farhang Radjai, Stéphane Roux. Friction-induced self-organization of a one-dimensional array of particles. Physical Review E , 1995, 51, pp.6177 - 6187. 10.1103/PhysRevE.51.6177 . hal-01425278

\section{HAL Id: hal-01425278 \\ https://hal.science/hal-01425278}

Submitted on 3 Jan 2017

HAL is a multi-disciplinary open access archive for the deposit and dissemination of scientific research documents, whether they are published or not. The documents may come from teaching and research institutions in France or abroad, or from public or private research centers.
L'archive ouverte pluridisciplinaire HAL, est destinée au dépôt et à la diffusion de documents scientifiques de niveau recherche, publiés ou non, émanant des établissements d'enseignement et de recherche français ou étrangers, des laboratoires publics ou privés. 


\title{
Friction-induced self-organization of a one-dimensional array of particles
}

\author{
Farhang Radjai \\ Höchstleistungsrechenzentrum, Forschungszentrum, 52425 Jülich, Germany \\ Stéphane Roux \\ Laboratoire de Physique et Mécanique des Milieux Héterogènes, \\ Ecole Supérieure de Physique et Chimie Industrielles de Patis, 10 rue Vauquelin, F-75291 Paris Cedex 05, France
}

\begin{abstract}
We study the frictional motion of a linear array of parallel identical cylinders on a plane where all interparticle and particle-plane contacts can be described by a Coulomb friction law. The entire system can be characterized in its steady state by an effective coefficient of friction and an effective inertia, both varying with the external driving force. In the steady-state regime, the organization of the array appears as regioos iovolving different rotation modes (sliding with no rotation, rolling on the support, alternate rotations, etc.). The mechanisms leading to this self-organization in modes are studied aualytically.
\end{abstract}

\section{INTRODUCTION}

In the context of standard continuum mechanics, the only field needed to describe the elastic deformation of a solid body is the displacement field alome. Rotations do not appear. They can be computed indirectly from the rotational of the displacement field. Other elasticity theories have been developed in order to account for the rotational degrees of freedom of a sohd material, such as "Cosserat" or "micropolar" theory [1]. Dimensional analysis shows that an intrinsic length scale $\lambda$ has to be introduced. This length may be interpreted as the scale below which rotations can significantly differ from those deduced from the rotation of the displacement field. At a larger scale, the difference vanishes, and thus upon coarse graiming the standard theory is recovered.

In the case of granular materials, the discontinuous geontetry of the solid phase, and thus of the displacement field, makes it difficult to estahlish a direct connection between a discrete description at the particle level and a continum homogenization at a large scale. In particular, the effect of individual rotations appears to play a determining role [2]. Although the framework of Cosserat theory seems more suited to such media, it does not appear to provide a simple key to the analysis. Some attempts in this direction have been proposed [3]; however, basic questions remain, such as the identification of this intrinsic length scale, which is often postulated to be a typical particle size. Other effects may motivate the study of rotations in granular media, such as the propagation of sound $[5,6]$.

*Also at Laboratoire de Physique et Mécanique des Milieux Héterogènes, ESPCI, 10 rue Vauquelin, F-75231 Paris Cedex 05, France.
Considering only the rotations of particles, one major difficulty appears immediately: the generally disordered geometry of the packing of particles induces a "frustration" of rotations. To mention only the two-dimensional case, as soon as a "loop" of contacting particles contains an odd number of elements, rotations of the particles in the loop are not possible without friction being mobilized on at least one contact. We employ the word frustration in analogy to the physics of spin glasses, where the presence of antiferromagnetic coupling between neighbors makes it impossible to satisfy all couplings simultaneously. We will not try here to develop the comparison between these two fields any further, although some suggestions along these lines have been proposed [4].

In this paper we consider a very simple system consisting of an array of disks in mutual contact supported by a plane. Two neighboring particles are in contact with the plane and thus a pair of particles and the support form a loop of three solids such that rotations are frustrated. Since frustration involves sliding on at least one contact, it is important to incorporate the friction law accurately in the description of the system. We will consider here the most basic law of friction, namely, Coulomb's law of friction.

When the array is pushed in the direction of its mean orientation, particles will move and rotate and a collcctive organization of rotations will emerge. This organization is rather complex. In particular, it involves typical length scales which may be much larger than the particle size, depending on the coefficients of friction (interparticle and particle-plane) and the confining pressure. This observation might motivate revisiting discrete-continuum transition through the analysis of intrinsic length scales different from the particle size.

In order to present our approach, we will first describe in Sec. II the simple case of a single particle being pushed on a plane. In Sec. III, we will consider the more general case of a long array and report on some numerical 
simulations. In Sec. IV, we will study analytically the collective organization of the array and describe the different phases observed. We will discuss the transition hetween the different phases and the expected effects on the global motion of the entire system.

\section{THE CASE OF A SINGLE PARTICLE}

Consider first the geometry illustrated in Fig. 1: A single disk supported by a basal plane is pushed by a constant horizontal force $N$. The mass and radius of the particle are assumed to be unity. The acceleration of gravity is set to unity as well. All quantities are normalized with respect to these natural units of the problem and, is particular, the moment of inertia $I$ is a mere geometric constant. The friction between the particle and the support is assumed to obey Coulomb's law with a friction coefficient $\mu^{\prime}$. The friction between the particle and the pushing block also follows Coulomb's friction law with a coefficient $\mu$. The translational and angular velocities of the particle are called $v$ and $\omega$, respectively. We are interested in the steady-state regime, where the acceleration is constant. All solids are assumed to be rigid, and thus they cannot interpenetrate. Figure 1 gives the convention used for the orientation of the forces. The tangent force at the particle-block contact is called $T$, whereas the friction force on the support is $S$. The normal reaction of the support is $R$.

The equations of the dynamics are the following:

$$
\begin{aligned}
\dot{U} & =N-S, \\
0 & =R-1-T, \\
I \dot{\omega} & =S-T,
\end{aligned}
$$

where the first two equations refer to Newton's law of motion in the horizontal and vertical directions, and the last one accounts for the rotation.

To find a complete solution to the equationg of dynamics, we need two more equations prescribed by the friction law at the two contact points. A friction law is a

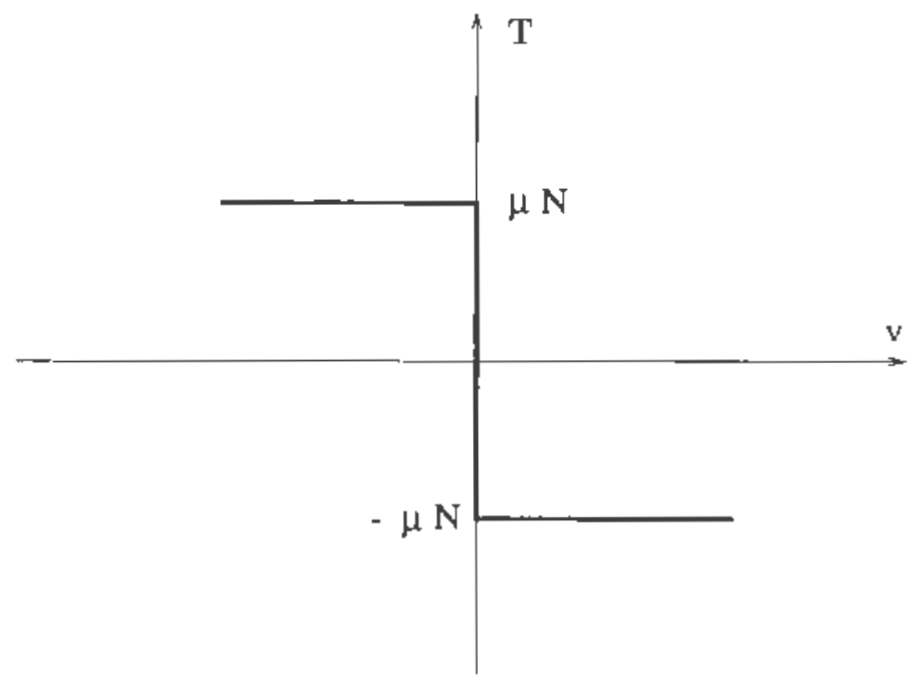

FIG. 1. Geometry of the single-sphere problem. relation between the friction force and the relative tangential velocity at the contact point between two solids. The graph of this relation for Coulomb's law is displayed in Fig. 2. In two dimensions, it is written

$$
\begin{aligned}
& v_{r}=0 \Rightarrow T \in[-\mu N, \mu N], \\
& v_{r}>0 \Rightarrow T=-\mu N, \\
& v_{r}<0 \Rightarrow T=\mu N,
\end{aligned}
$$

where $v_{r}$ is the relative tangential velocity and $T$ and $N$ are the tangential and normal components of the contact force. In the case where $v_{r}=0$, there is no equation relating the tangential force to the normal contact force. In the present case of a single particle, we will consider different configurations with different relative velocities at the contact points and we will show that the friction force is always uniquely determined. In the next section, we will discuss a more general formulation of Coulomb's law adapted to dynamical systems [7].

There are two particular values of rotation velocities: $\omega_{1}=0$ and $\omega_{2}=v$. Depending on the relative position of $\omega$ with respect to these particular values, the tangent force at the contacts will assume different values. However, up to a change in the origin of time, the position of $\omega$ with respect to the key values $\omega_{i}$ can he equivalently analyzed through the relative positions of the accelerations $\dot{\omega}$ with respect to $\dot{\omega}_{i}$, since we are interested in the steady state, where the accelerations remain constant in time. Five different cases can be distinguished.

(1) $\dot{\dot{y}}>\dot{v}$. In this case, we have $T=\mu N$ and $S=$ $-\mu R$. Since both tangent forces, $T$ and $S$, contribute to a torque opposite to $\omega$, the angular acceleration has to turn negative. Therefore, no steady-state solution can be found.

(2) $\dot{\omega}=\dot{v}$. In this case, $T=\mu N$, but $S$ cannot be deduced directly from Coulomb's law. However, the kinematic constraint $\dot{\omega}=\dot{v}$ suffices to determine the system entirely. The complete solution is written

$$
\begin{array}{ll}
T=\mu N, & \dot{v}=\left(\frac{1-\mu}{1+I}\right) N, \\
R=I+\mu N, & \\
S=\left(\frac{I+\mu}{I+1}\right) N, & \dot{\omega}=\left(\frac{I-\mu}{I+I}\right) N .
\end{array}
$$

For this solution to be acceptable, the condition $|S|<$ $\mu^{\prime} R$ has to be satisfied. This gives a constraint on the value of $N$ :

$$
N<N_{1}=\frac{\mu^{\prime}(1+I)}{I+\mu-\mu \mu^{\prime}(1+I)} .
$$

(3) $0<\dot{\omega}<\dot{v}$. In this case, $T=\mu N$ and $S=\mu \mu^{\prime} R$ as given by Eq. (2). The complete solution of the system is

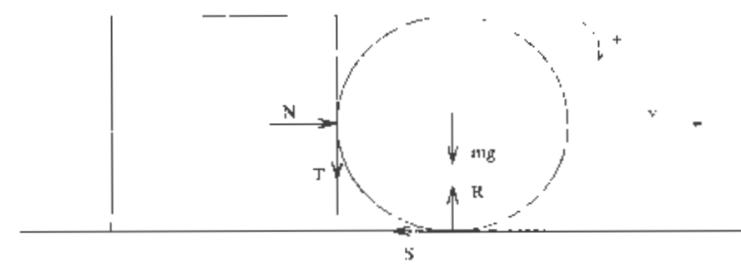

FIG, 2. Coulomb's law graph. 


$$
\begin{array}{ll}
T=\mu N, & \dot{v}=-\mu^{\prime}+\left(1-\mu^{\prime} \mu\right) N \\
R=1+\mu N, & \dot{\omega}=\frac{1}{\Gamma}\left(\mu^{\prime}-\left(1-\mu^{\prime}\right) \mu N\right) \\
S=\mu^{\prime}(1+\mu N), &
\end{array}
$$

Compatibility of this solution with the conditions $0<$ $\dot{\omega}<\dot{v}$ sets the following constraints on the values of $N$ :

$$
N_{1}<N<N_{2}=\frac{\mu^{\prime}}{\mu\left(1-\mu^{\prime}\right)}
$$

(4) $\dot{\omega}=0$. In this case, $S=\mu^{\prime} R$ while $T$ is not determined by the friction law directly. The condition $\dot{\omega}=0$, however, gives an additional constraint which leads to

$$
\begin{aligned}
& T=\frac{\mu^{\prime}}{1-\mu^{\prime}}, \quad \dot{v}=N-\frac{\mu^{\prime}}{1-\mu^{\prime}}, \\
& R=\frac{1}{1-\mu^{\prime}}, \quad \dot{\varphi}=0 . \\
& S=\frac{\mu}{1-\mu^{\prime}},
\end{aligned}
$$

The condition $|T|<\mu N$ implies

$$
N>N_{2}
$$

(5) $\dot{\omega}<0$. In this case, $S=\mu^{\prime} R$ and $T=-\mu N$. As in the fist case, the two tangent forces contribute to a torque opposite to $\omega$ and thus no steady-state solution is possible.

In this way, the dynamics of the block-particle system show three possible steady-state phases: rolling (phase 1), rotating and sliding on the plane (phase 2), and sliding on the plane without rotating (phase 3). Figure 3 shows the friction force $S$ at the particle-plane contact as a function of the driving force $N$. The former increases linearly with the apphied force in the two first phases to saturate to a value greater than $\mu^{\prime}$ in the last one.

As long as the translational motion of the particle is of interest, these results can be compared to the dynamics of a rigid block of mass $m$, pushed by a force $N$. The respective equation of motion is written

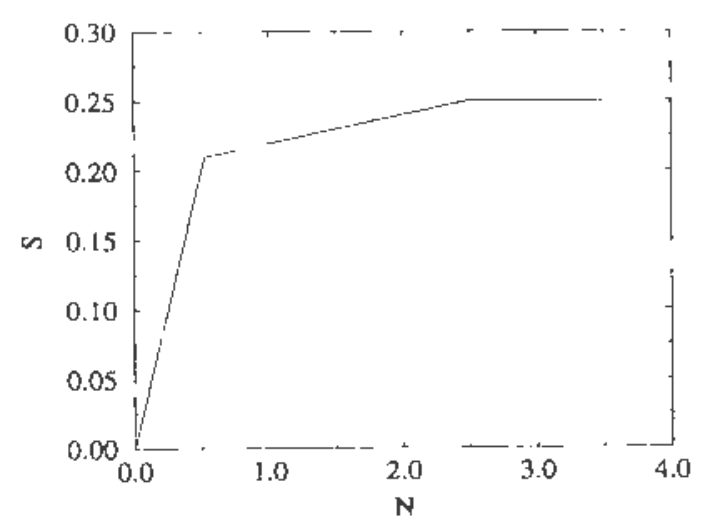

FIG. 3. Evolution of the particle-plane friction force as a function of the driving force $N$. All forces are normalized with respect to the weight of one particle (see text). The first regime $N<N_{1}$ corresponds to rolling on the support, the intermediate regime $N_{1}<N<N_{2}$ corresponds to partial rotation where both contacts are sliding contacts, and finally in the last regime $N>N_{2}$, the particle slides on the support with no rotation. Here, $\mu=0.1$ and $\mu^{\prime}=0.2$.

$$
N-M m=m \dot{v}
$$

where $M$ is the coefficient of friction at the block-plane contact. Now, a look at the steady-state solutions of the single-particle problem [Eqs. (3), (5), and (7)] in different regimes shows that they can all be written in the canonical form of Eq. (9) if we introduce an effective inertia $m^{*}$ and an effective coefficient of friction $M^{*}$ :

$$
m^{*}= \begin{cases}\frac{(1+J)}{(1-\mu)}, & N<N_{1} \\ \frac{1}{\left(1-\mu^{\prime} \mu\right)}, & N_{1}<N<N_{2} \\ 1, & N_{2}<N .\end{cases}
$$

The "effective friction force" on the plane is given by $M^{*} m^{*}$. While it is obvious that the inertia $m^{*}$ should depend on whether the particle slides or rolls, it is surprising that the coefficients of friction $\mu$ and $\mu^{\prime}$ enter the expression for the inertia. Note that the effective inertia is piecewise constant, and decreases by steps with $N$. Similarly, the effective coefficient of friction $M^{*}$ also depends on the driving force in a piecewise constant manner, increasing with $N$. The first case, which corresponds to a low applied force, is somewhat counterint uitive. The effective coefficient of friction is exactly zero, even though there is a nonzero friction force in the system. This friction force being proportional to the acceleration, it only contributes to the effective inertia rather than to the coefficient of friction $M^{*}$. We will see below that this behavior remains qualitatively valid for an array of particles, with essentially the same low force behavior (zero coefficient of friction).

The steady state can be characterized equivalently by the rotation of the particle. The relevant dimensionless variable is then $\alpha=\dot{\psi} / \dot{v}$. The steady-state diagram in the $N-\alpha$ space is shown in Fig. 4. For a given value of the driving force, whatever the initial velocities, $\alpha$ is attracted to a point on the steady-state diagram. In phase 1 , the steady-state value of $\alpha$ is equal to unity, while it is equal to zero in phase 3. In phase 2 , $\alpha$ decreases with the driving force as

$$
\alpha=\frac{1}{I} \frac{\mu^{\prime}-\left(1-\mu^{\prime}\right) \mu N}{\left(1-\mu \mu^{\prime}\right) N-\mu^{\prime}} .
$$

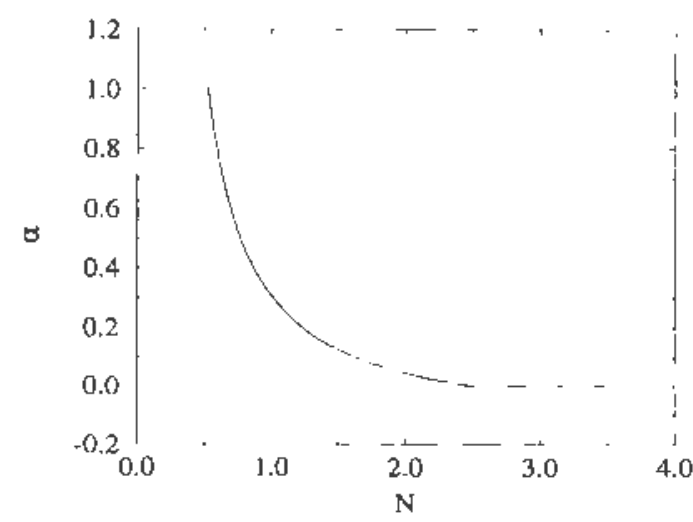

FIG. 4. Steady state of the single-particle problem in $N-\alpha$ space, where $\alpha=\frac{\dot{\omega}}{\dot{v}}$. All forces and accelerations are normalized (see text). The steady state depends only on parameters and not on initial conditions. Here, $\mu=0.1$ and $\mu^{\prime}=0.2$. 
It can also be shown that the particular motion of the particle, for a given driving force, is the one that minimizes the dissipation.

In the equations of the problem, the particular value of unity for the coefficients of friction appears to play a key role. So far, we have tacitly assumed that the two coefficients of friction were smaller than unity. However, it is now straightforward to understand what happens when this is not the case. If $\mu<1$ and $\mu^{\prime}>1$, then the only steady state is phase 1 : the particle can only roll on the plane. If $\mu>1$ and $\mu^{\prime}<1$, then it is phase 3 that dominates the steady state: the particle can only slide on the plane. In the case where both $\mu$ and $\mu^{\prime}$ are greater than unity, no motion at all is possible! The particle simply sticks to the plane. It is easy to see that if the driving force $N$ had a component downward, then the same situation would occur even for smaller values of the coefficients of friction. In this respect, unity has nothing special and reflects only the particular geometry we consider here. In a long array of particles this same effect takes place when the coefficients of friction are greater than unity, and the applied force is screened by the particle in contact with the pushing block. This ohservation suggests that in granular media, things will change radically when the interparticle coefficient of friction is high enough. In the forthcoming sections we will assume that the coefficients of friction are smaller than unity.

\section{A ONE-DIMENSIONAL ARRAY OF PARTICLES}

The study of the motion of a single particle provides us already with some insight into the question we address in the general case of a long array. How are the rotations of the particles orgamized in the steady state? How does the motion of the system depend on the applied force? Our aim is to propose a global descriptiou of the frictional motion of this system as a result of the collective organization of rotations.

We have set up a computer program to simulate the motions of particles on a plane. The case of a single particle (Sec. II) shows that the friction law is actually the most important ingredient of the problem. Our program prescribes the exact Coulomb law (with no regularization) at the particle-particle and particle-plane contacts. We are interested in the situation where each particle remains in contact with its neighbors, so that the whole system can be considered as a singlr object. This requirement is motivated by our purpose here to focus on the role played by the rotations in the translational motion of the array. Only if all interparticle contacts are closed can the global translation be defined. Hence, the system we simulate is collision free and the relative velocities at contact points are only tangential. Moreover, ow system involves no cohesion force and no elasticity.

As we shall see later in this section, the close-contact condition is satisfied only for a limited region in the parameter space. We add a second block to confine the array, where a constant force opposite to the direction of motion is applied to the first particle of the array. Although this new parameter is neither nccessary nor sufficient to prevent particle-particle contacts from opening, it is, in general, a very useful control parameter, especially when the driving force is weak. So, the parameters are the following: $\mu, \mu^{\prime}$, and $\mu^{\prime \prime}$ are the coefficients of interparticle, plane-particle, and block-particle friction, respectively. The driving force $N_{L}$, the confining force $N_{0}$, and the number of particles $L$ are the other parameters, as shown in Fig. 5 .

\section{A. Governing equations}

Figure 5 shows a scheme of the array with the sign conventions for forces used throughout this paper. With these conventions, the equations of dynamics for each particle $i$ are written

$$
\begin{aligned}
T(i)-T(i-1)+R(i) & =1 \\
N(i)-N(i-1)+S(i) & =\dot{v} \\
T(i)+T(i-1)-S(i) & =I \dot{\omega}(i) .
\end{aligned}
$$

The houndary conditions are

$$
\begin{aligned}
& N(L)=N_{L}, \\
& N(0)=N_{0} .
\end{aligned}
$$

There are $5 L+1$ variables to be determined, whereas we have only $3 L$ equations given by dynamics. The remaining $2 L+1$ equations are prescribed by the contact law at the $2 L+1$ contact points.

In order to avoid any artifact in the solution of our equations, we would like to use the exact Coulomb law of friction. However, it is not straightforward to inplement it in an algorithm, since, as shown in Fig. 2, the $\left(v_{r}, T\right)$ should belong to a set of admissible values.

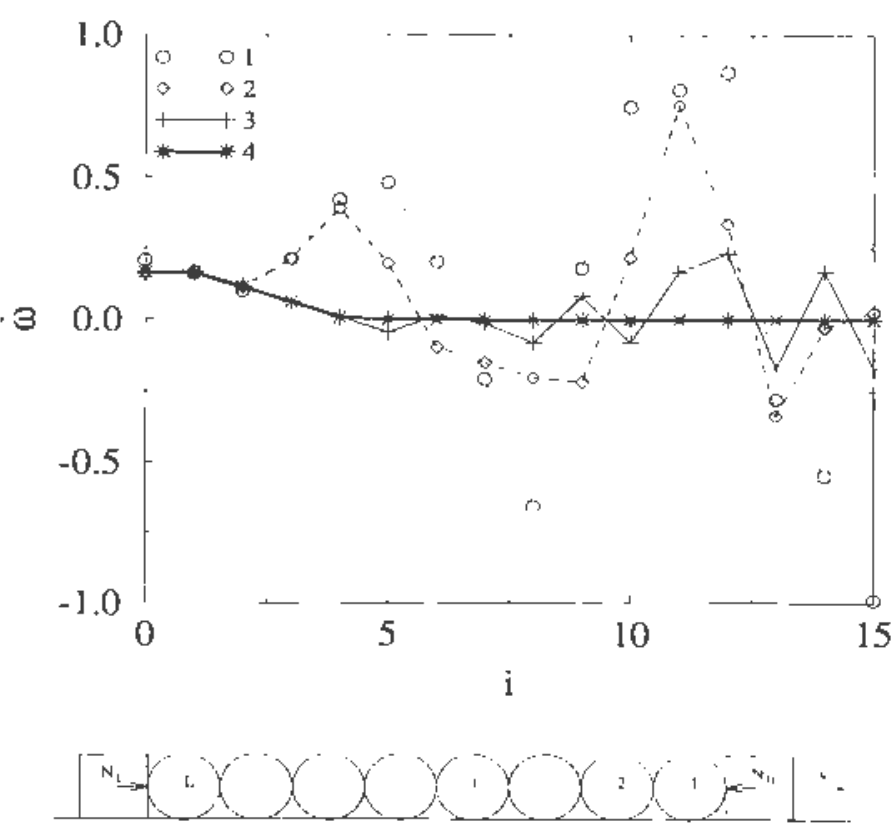

FIG. 5. (a) An array of particles on a plane. (b) Forces exerted on the particle $i$ and sign conventions. 
A mathematical framework for handling such problems, known as nonsmooth analysis, has been developed [8], In static equilibrium, where all the relative velocities are zero, Coulomb's law results indeed in an indeterminate state of forces (the so-called hyperstaticity of a sandpile at equilibrium). Nevertheless, in a dynamical system where the particles are moving and the states of contacts are permanently subject to change, the friction force on the vertical branch of Coulomb's law can be determined in a unique way. We have shown this explicitly in the case of the single-particle problem. The main point is that one has to distinguish the contacts where the relative tangential velocity remains equal to zero from those where this is not so. In terms of the relative accelerations, we would say that there are two classes of contacts where the relative tangential velocity is zero: one where only the relative tangential velocity is equal to zero and one where both the relative tangential velocity and acceleration are equal to zero. We shall call them active contacts and nonsliding contacts, respectively. Whenever a contact is active, i.e., when $v_{r}=0$, there are three different alternatives distinguished by the relative tangential acceleration $\dot{v}_{r}$ at the contact.

(1) $\dot{v}_{r}=0$. In this case, the contact is nonsliding. The friction force $T$ has to be in Coulomb's limits $[-\mu N, \mu N]$, where $N$ is the normal force at the contact. Here we have an equaiton and an inequality. When the equation $\dot{v}_{r}=0$ supplements the equations of dynamics, we get the values of $T$ and $N$ and we can check for the inequality. If the inequality is satisfied as well, then we have the solution. If this is not so, we have to switch to one of the two other alternatives.

(2) $\dot{v}_{r}>0$. In this case, the contact is sliding although it is active. So, we have to put $T=-\mu N$ following the sign convention of Fig. 2. We supplement the equations of dynamics with the latter equation, from which we calculate $\dot{v}_{r}$ among others, and we check for the inequality. If the inequality is not satisfied, then we turn to another alternative.

(3) $\dot{\nu}_{r}<0$. This is the same as the second case except that the corresponding equation is $T=\mu N$.

In this way, Coulomb's law in its most general formulation takes the following form:

$$
\begin{aligned}
& v_{r}=0 \Rightarrow\left\{\begin{array}{l}
\dot{v}_{r}=0 \text { and } T \in[-\mu N, \mu N], \\
\dot{v}_{r}>0 \text { and } T=-\mu N, \\
\dot{v}_{r}<0 \text { and } T=\mu N,
\end{array}\right. \\
& v_{r}>0 \Rightarrow \quad T=-\mu N, \\
& v_{r}<0 \Rightarrow T=\mu N .
\end{aligned}
$$

In order to solve the system of equations when a contact becomes active, the three alternative configurations are to be tried successively until the solution is found. Actually, there may be a great number of nonsliding contacts in a granular medium at the same time. Whenever a single contact hecomes active, all the other nonsliding contacts are to be considered as well. For $p$ active contacts, this implies $3^{p}$ possible configurations. Depending on the situation, different phenomena may take place. When a contact becomes active, it may become nonslid- ing, but cause other nonsliding contacts to turn shiding at the same time; or it may simply turn out to be a sliding contact and force other nonsliding contacts to become sliding ones as well, and so on.

This basic image is too time consuming to be implemented in an algorithm, since the number of possible configurations grows exponentially with the number of active contacts. An important ingredient of our computer program is a relaxation scheme that converges rapidly to the solution in the space of configurations. The method used is to start with an arbitrary configuration when, in the process of evolution of the system, some contact becomes active. Then, if the selected configuration is not the right solution, it is mapped onto the space of configurations in order to build a new configuration to be tested. This iterative process converges very rapidly to the solution with the following mapping:

$$
\begin{array}{llll}
\dot{v}_{r}=0 \text { and } T<-\mu N & \longrightarrow & T=-\mu N, \\
\dot{v}_{r}=0 \text { and } T>+\mu N & \longrightarrow & T=+\mu N, \\
T=-\mu N \text { and } \dot{v}_{r}<0 & \longrightarrow & \dot{v}_{r}=0, \\
T=+\mu N \text { and } \dot{v}_{r}>0 & \longrightarrow & \dot{v}_{r}=0 .
\end{array}
$$

This procedure has to be applied simultaneously to all active contacts.

The evolution of the system can be studied starting from a random initial state. The easiest way to generate initial conditions is to choose initial velocities in such a way that all contacts are shiding. For a given value of the driving force $N_{L}$, all accelerations and forces can then be determined. These values do not change unless some contact becomes active. Meanwhile, the motion of each particle is uniformly accelerated and the time $\delta h$ needed for some contact to become active is simply given by

$$
\delta h=\min \left\{\delta h(i)=-\frac{v_{r}(i)}{\dot{v}_{\Gamma}(i)}, \quad \delta h(i)>0\right\} .
$$

At this moment, a relative tangential velocity at some contact vanishes and new values of forces and accelerations are to be calculated. Since the states of some contacts change, the values of forces and accelerations undergo discontinuous changes. The motion is again uniformly accelerated. This event-driven process continues until the system achieves the steady state where at every contact the relative velocity and acceleration are of the same sign or both are zero. In the steady state, all forces and accelerations remain the same forever.

\section{B. Numerical results}

Onr simulations show that, depending on the driv ing force, three different regimes may occur. For smali enough values of the driving force and for most initial conditions, some interparticle contact opens and the array separates in two or more identical arrays. On the other hand, for large enough values of the driving force, some particle-plane contact may be lost and the geometry of the array is modified because of the rising of one particle. In between these two limits, all contacts are preserved. We consider only this intermediate regime, 
where the driving force can be varied without causing any modification of the geometry.

In this case, the system evolves to a steady state that is independent of the initial conditions (i.e., initial velocities) for a given set of parameters. Figure 6 shows the angular accelerations of particles in an array of 15 particles at four stages of the evolution of the system. The acceleration corresponding to the coordinate 0 stands for the translational acceleration of the array. For different initial conditions, the transient stage differs from one simulation to another, but the final state of motions is always the same. The steady-state forces and accelerations are only a function of the boundary conditions and parameters and do not depend on the evolution.

Figure 7 shows the steady-state forces and accelerations for an array of 40 particles. Different motion patterns appear successively along the array. A few particles, starting from the particle in contact with the confining block, just roll withont sliding on the plane. This mode of rotation will be referred to as phase 1 . After that, we have particles with positive rotation velocities and which slide at the same time on the plane. In this phase, which will be referred to as phase 2, all of the contacts are sliding contacts. Next, we have particles rotating in opposite directions and sliding on the plane. In this phase, to which we will refer as phase 3 , all interparticle contacts are nonsliding.

This succession of phases 1,2 , and 3 along the array is the most typical pattern of rotation emerging from any initial condition. It changes only quantitatively with the parameters, so that the length of each phase may vary and, for high enough values of the driving force, phase 1, phase 3 , or phases 1 and 2 may simply disappear. In the same way, the angular rotations in phase 3 may vanish. We will come back to these patterns in Sec. IV.

Let us now consider the linear acceleration of the array on the plane. A "global coefficient of friction," $M$, can be defined for this system by the total friction force on the plane $N_{L}-N_{0}-L \dot{v}$ divided by the total weight $L$ :

$$
M=\frac{N_{L}-N_{0}}{L}-\dot{v} \text {. }
$$

The evolntion of $M$ for an array of 20 particles is displayed in Fig. 8 for eight different simulations with the same parameters, but with different initial conditions. The steady-state value of $M$ is the same, although the intermediate values before reaching the steady state are different in each case. In other words, $M$ characterizes the global friction of the array on the plane in a unique way. In the simulations of Fig. 8 , the values of parame-

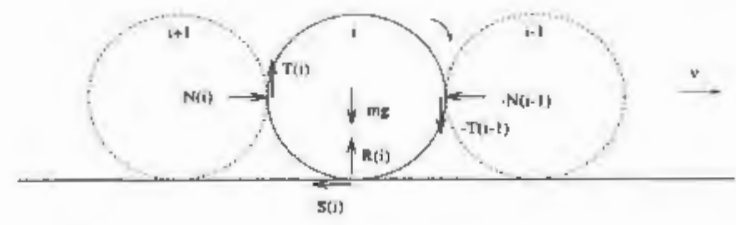

FIG. 6. Evolotion of the angular accelerations of particles in an array of 15 particles. The acceleration corresponding to the coordinate 0 stands for the translational acceleration $\dot{v}$. In this simulation, $\mu=\mu^{\prime \prime}=0.05$ and $\mu^{\prime}=0.1$.
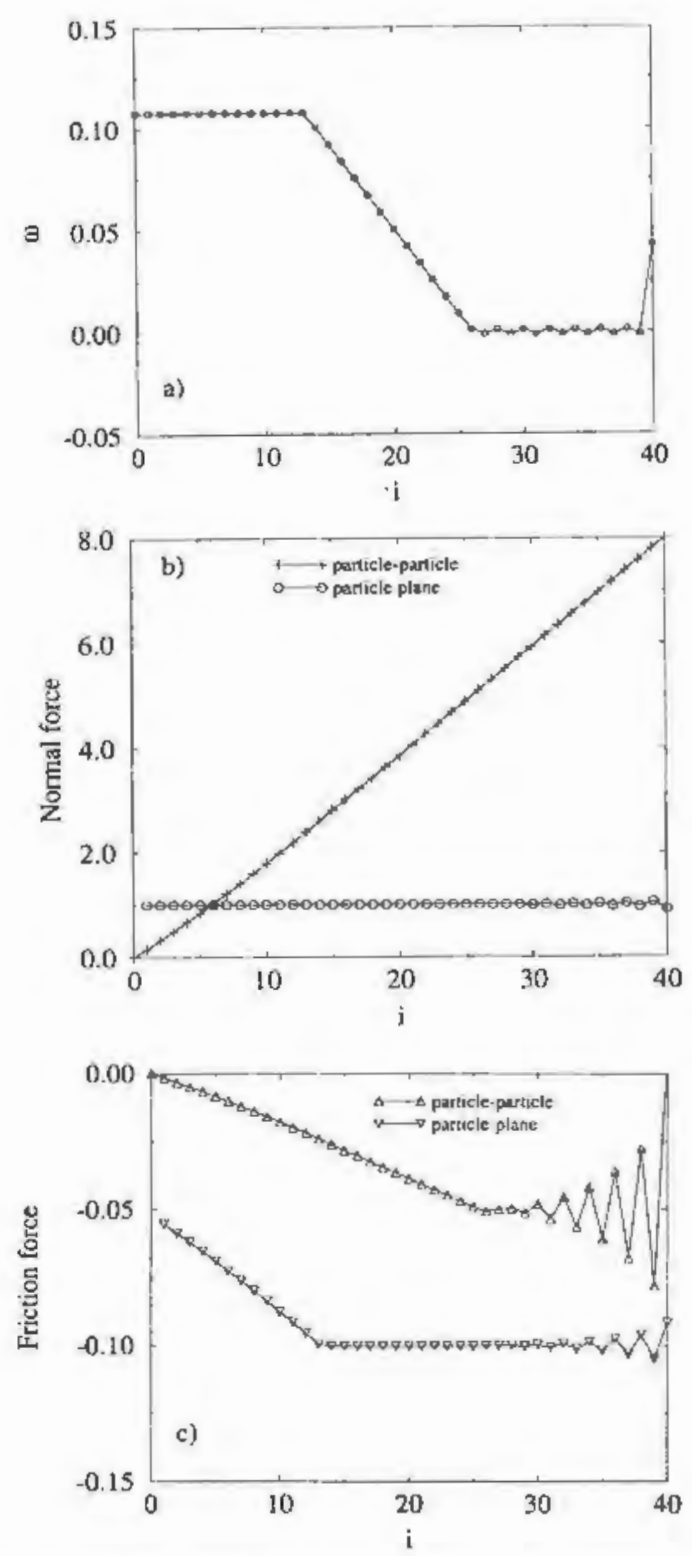

FIG. 7. Accelerations and forces along an array of 40 particles. (a) Accelerations. The acceleration on coordinate 0 stands for the translational acceleration $\dot{v}$. (b) Normal forces at the contact points. (c) Tangent friction forces at contact points. Parameters are $N_{L}=8.0, \mu=0.01, \mu^{\prime}=0.10$, and $N_{0}=0$.

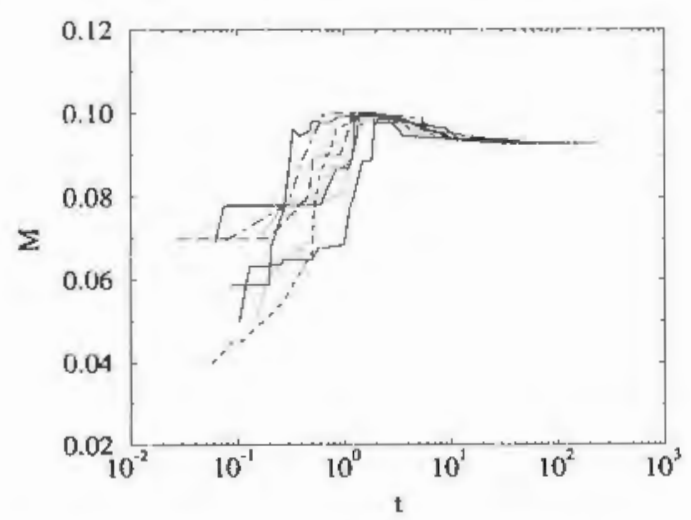

FIG. 8. Evolution of the global coefficient of frictiou $M$ for eight different simulations of the same system beginning with different initial conditions. Parameters are $\mu=0.01, \mu^{t}=0.1$, $N_{L}=4.5$, and $N_{0}=0.05$. 
ters are such that the steady-state value of $M$ is smaller than the particle-plane coefficient of friction.

The global steady-state coefficient of friction $M$ of the array, although independent of the initial conditions, differs from the ordinary coefficient of friction in that it is a function of the driving force. Figure 9 shows this dependence for a system of 20 particles. $M$ increases from zero linearly with $N_{L}$ and saturates to a value equal to the particle-plane coefficient of friction. In other words, for weak values of $N_{L}$, the driving force is accelerating the system less than it would if the particles were replaced by a single block. Another effect of the driving force is to regulate the internal organization of forces so as to increase its frictional resistance. For weak enough values of the driving force, the system is in phase 1. Dissipation takes place only at interparticle contacts. In this regime, $M$ increases linearly with the driving force hecause, as we shall see in the next section, in phase 1 all forces scale with the driving force except for the normal force on the plane. As the driving force increases, more and more particles of phase 2 appear in the tail of phase 1 and hence the number of particles in phase 1 decreases. Then, $M$ is no more linear in $N_{L}$. Finally, when the driving force is high enough, phase 1 disappears completely from the array so that all particles are either in phase 2 or in phase 3 . In this regime, $M$ is simply equal to the particle-plane coefficient of friction. Thus, the number of rolling particles indicates the capacity of the system to increase its friction. Figure 10 shows the configuration of rotations in the steady state of a system of 40 particles for different values of the driving force. The connection between the rotation modes and the global behavior of the array in translation will be studied in more detail in the next section.

\section{THEORETICAL ANALYSIS OF THE STEADY STATE}

In this section we will focus on the steady state and the mechanisms leading to the spatial patterns of rotations. These patterns provide the key to the global description of the array as a single object in displacement.

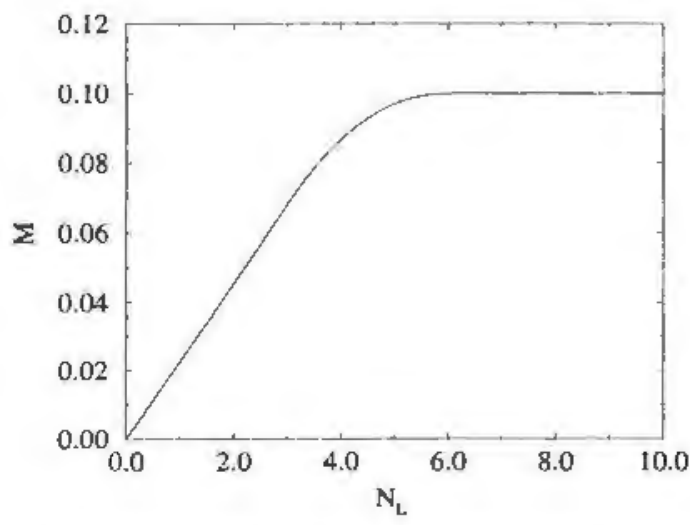

FIG. 9. Variation of the global coefficient of friction of an array of 20 particles as a function of the driving force. $\mathrm{Pa}$ rameters are $\mu=0.01, \mu^{\prime}=0.1$, and $N_{0}=0.05$.

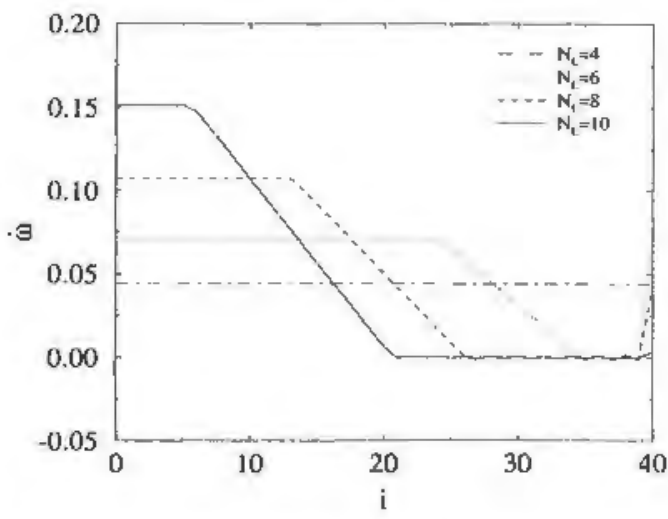

FIG. 10. Angular accelerations of particles in an array of 40 particles for different values of the applied force. Here, $\mu=0.01, \mu^{\prime}=0.10$, and $N_{0}=0$.

\section{A. Different phases}

The most interesting feature of the rotation patterns is that pure phases of rotation come one after another without mixing. This scheme suggests that as one goes along the array, some quantitative changes in the state of forces are followed by qualitative changes resulting in quite different behaviors. Let us consider first the case of rolling (phase 1).

Since all of the particles in phase 1 are rolling in the same direction, friction forces at interparticle contacts are fully mobilized so that the equations of dynamics can be supplemented in this case by

$$
T(i)=-\mu N(i)
$$

Moreover, rolling implies

$$
\dot{\omega}(i)=\dot{v} .
$$

From Eqs. (12), (18), and (19) we get the following expression for the friction force at particle-plane contacts:

$$
S(i)=\dot{v}-\frac{2 \mu}{1+\mu}\left(N_{0}+\frac{1+I}{2 \mu} \dot{v}\right)\left(\frac{1+\mu}{1-\mu}\right)^{i}
$$

as well as the following limit on the value of the particleplane force:

$$
-S(i) \leq \frac{\mu^{\prime}(1+\mu \dot{v})}{1+\mu \mu^{\prime}}
$$

Equation (20) shows that the particle-plane friction is mobilized progressively along the array and its direction is opposite to that of the motion. The absolute value of the friction force has an upper bound given by (21). From (20) and (21) one gets the maximum number of particles in phase 1:

$$
\begin{aligned}
i \leq L_{1}=1 & +\left\{\ln \left(\frac{1}{2 \mu} \frac{1-\mu}{1-\mu \mu^{\prime}}\left(\dot{v}+\mu^{\prime}\right)\right)\right. \\
& \left.-\ln \left(N_{0}+\frac{1+I}{2 \mu} \dot{v}\right)\right\} / \ln \left(\frac{1+\mu}{1-\mu}\right),
\end{aligned}
$$


where $L_{1}$ is the length of phase 1 . So, the simple mechanism behind this phase is the progressive mobilization of the friction force at particle-plane contacts up to the maximum possible value of the friction force. Simulations in fact confirm this simple picture; see the functions $S(i)$ and $\dot{\omega}(i)$ in Fig. 7 from the simulation of an array of 40 particles. The contacts of the particles coming after the particle $i=L_{1}$ with the plane can no longer be nonslidirig and in phase 2 , even if the particles continue rotating in the same direction, all contacts are sliding contacts.

Now, let us consider the situation where all contacts are sliding contacts and all of the particles rotate in the positive direction (phase 2). The following equations are then to be used to supplement the equations of dynamics:

$$
\begin{aligned}
& T(j)=-\mu N(j) \\
& S(j)=-\mu^{\prime} R(j) \\
& \dot{\varphi}(j)>0 .
\end{aligned}
$$

From these and the equations of dynamics we get the following expression for the angular accelerations:

$$
\begin{aligned}
I \dot{\omega}(j)= & -\frac{2 \mu\left(\mu^{\prime}+\dot{v}\right)}{1-\mu \mu^{\prime}} j+\frac{\mu\left(1+\mu^{\prime}\right)}{1-\mu \mu^{\prime}} \dot{v} \\
& +\frac{\mu^{\prime}(1+\mu)}{1-\mu \mu^{\prime}}-2 \mu^{\prime} N(j=0),
\end{aligned}
$$

where $j$ refers to the $j$ th particle of phase 2 and $N(j=0)$ is the normal interparticle force on the first partricle in the phase. At the same time, we have

$$
S(j)=-\frac{\mu^{\prime}(1+\mu \nu)}{1-\mu \mu^{\prime}}
$$

These equations show that the angular acceleration is decreasing linearly with the particle number while the friction force at the particle-plane contact remains constant. However, the positivity of the angular acceleration, which is a consistency condition, gives the maximum number of particles in the phase:

$$
\begin{aligned}
j \leq L_{2}=\frac{1}{\mu^{\prime}+\dot{v}} & {\left[\frac{1+\mu^{\prime}}{2} \dot{v}+\frac{\mu^{\prime}(1+\mu)}{2 \mu}\right.} \\
& \left.-\left(1-\mu^{\prime}\right) N(j=0)\right]
\end{aligned}
$$

where $L_{2}$ is the length of phase 2. This simple mechanism behind phase 2 is illustrated in Fig. 7 , where $T(i)$ and $\omega(i)$ are shown for a sirnulation of an array of 40 particles. From the end of phase 2, the behavior changes again radically as the particles can no longer rotate consistently in the same direction.

Let us now consider phase 3 , where the interparticle contacts can no longer be sliding. The equations to be used to supplement the equations of the dynamics are the following:

$$
\begin{aligned}
& S(k)=-\mu^{\prime} R(k), \\
& \dot{\omega}(k)=-\dot{\omega}(k+1)=\dot{\omega} .
\end{aligned}
$$

Assuming that the first particle in this phase in contact with the last particle of phase 2 has a negative rotation velocity, we immediately get the following expression for the tangential interparticle force:

$$
\begin{aligned}
T(k)= & {\left[T(k=0)+\frac{\mu^{\prime}}{2}+\frac{I \omega}{2 \mu^{\prime}}\right]\left(\frac{1+\mu^{\prime}}{1-\mu^{\prime}}\right)^{k} } \\
& -(-1)^{k} \frac{I \omega}{2 \mu^{\prime}}-\frac{\mu^{\prime}}{2} .
\end{aligned}
$$

The interparticle friction force oscillates with a period of two particles and grows exponentially. We renıark that this behavior of the interparticle friction force is only related to the fact that the interparticle contacts here are nonsliding. Even in the case where $\dot{w}=0$, which is a particular case of phase 3 , this oscillating behavior is obtained. In the same way, we get the following expression for the normal interparticle force:

$$
\begin{aligned}
N(k)= & N(k=0)+k\left(\dot{v}+\mu^{\prime}\right) \\
& +\mu^{\prime}\left[T(k=0)+\frac{\mu^{\prime}}{2}+\frac{I \dot{\omega}}{2 \mu^{\prime}}\right] \\
& \times\left[1-\left(-\frac{1+\mu^{\prime}}{1-\mu^{\prime}}\right)^{k}\right]
\end{aligned}
$$

From Eqs. (28) and (29) and the boundary condition $T(0)=-\mu N(0)$, it is easy to see that $|T(k)| \leq \mu N(k)$ for all $k$. The length of phase 3 is thus not limited by the mobilization of the interparticle friction forces. Howcver, some limit arises from the normal particle-plane force which, for consistency of the equations, has to be positive. The expression of the normal particle-plane force is the following:

$$
\begin{aligned}
R(k)= & 1+(-1)^{k} \frac{I \dot{\omega}}{\mu^{\prime}} \\
& +\frac{2}{1-\mu^{\prime}}\left[T(k=0)+\frac{\mu^{\prime}}{2}+\frac{I \dot{\omega}}{2 \mu^{\prime}}\right] \\
& \times\left[-\frac{1+\mu^{\prime}}{1-\mu^{\prime}}\right]^{k-1} .
\end{aligned}
$$

The positivity of $R(k)$ sets an upper limit on the length of phase 3:

$$
\begin{aligned}
k \leq L_{3}=1 & +\left\{\ln \left[\frac{1-\mu^{\prime}}{2}\left(1+\frac{I \dot{\omega}}{\mu^{\prime}}\right)\right]\right. \\
& \left.-\ln \left[T(0)+\frac{\mu^{\prime}}{2}+\frac{I \dot{\omega}}{2 \mu^{\prime}}\right]\right\} / \ln \left(\frac{1+\mu^{\prime}}{1-\mu^{\prime}}\right),
\end{aligned}
$$

where $L_{3}$ is the maximum length of the phase and $T(0)$ is to be replaced with the last particle in phase 2. The particle coming just after the particle $L_{1}+L_{2}+L_{3}$ will rise as the reaction force on the plane vanishes.

What is the relation between $\dot{\omega}$ and $\dot{v}$ in phase 3 ? There are not enough equations to relate these two accelerations. The answer is to be looked for in the bonndary conditions. For the array to be stable (no contact opening), we should have $L \leq L_{1}+L_{2}+L_{3}$. Suppose that there is an even number of particles in phase 3 so that the last particle is rotating in the positive direction. As the latter is in contact with a block, with a coefficient of 
friction generally different from $\mu$, its angular acceleration is different from that of phase 3. Simulations show that if $\mu^{\prime \prime}<\mu$, then the angular acceleration of the last particle is greater than that of phase 3 . In this case, the contact between the last particle of the array and the last particle of phase 3 is sliding and we have the consistency condition $T\left(k=L_{3}^{\prime}-1\right)=-\mu N\left(k=L_{3}^{\prime}-1\right)$, where $L^{\prime}$ is the length of phase 3 . This gives the relation between $\dot{\omega}$ and $\dot{v}$. As seen in Fig. 10, $\dot{\omega}$ decreases with the driving force, while $\dot{v}$ increases. Figure 11 shows the angular accelerations of the particles in two simulations: one with $\mu^{\prime \prime}=0$, the other with $\mu^{\prime \prime}>\mu$. In this latter case, the rotations in phase 3 simply vanish.

\section{B. Global dynamics}

The global behavior of the array results from the juxtaposition of the three rotation phases. By global bebavior, we mean the relation between the applied force $N_{L}-N_{0}$, as input to the system, and the acceleration $\dot{v}$, which is a global output. In this section we will show that, as in the case of a single particle (Sec. II), this relation can be cast in the canonical form of the equation of frictional motion of a single block:

$$
N_{L}-N_{0}-F^{*}=m^{*} \dot{v},
$$

where $m^{*}$, the effective inertia and $F^{*}$, the effective friction force on the plane, are functions of the parameters and the applied force. Figure 12 shows $\dot{v}$ as a function of $N_{L}-N_{0}$ for an array of 20 particles. The inverse of the slope at each point corresponds to the effective inertia for the corresponding value of the applied force, while the intersection with the axis of accelerations gives the effective friction force on the array. Three different regimes can be distinguished.

(1) From zero up to a critical value $N^{\prime}$ of the applied force, the effective mass is constant. In this regime all particles are in phase 1. From Eqs. (12), (18), and (19) it can be shown that the normal interparticle force in phase 1 is given by

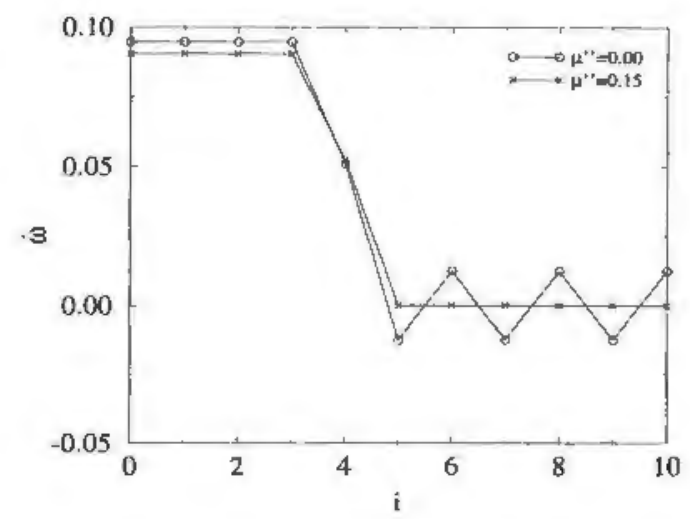

FIG. 11. Angular accelerations of particles in an array of ten particles for two different values of the particle-block coefficient of friction $\mu^{\prime \prime}$. The other parameters are $\mu=0.1$, $\mu^{\prime}=0.2, N_{0}=0.1$, and $N_{L}=2.8$.

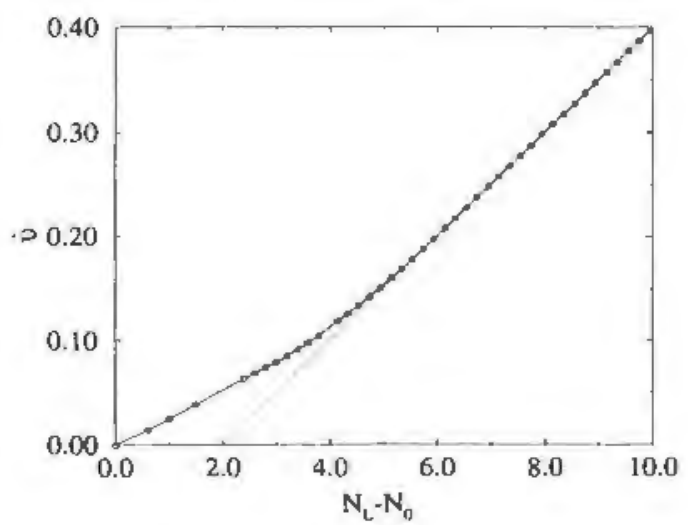

FIG. 12. Translational acceleration of an array of 20 particles as a function of the applied force $N_{L}-N_{0}$. Parameters are $\mu=0.01, \mu^{\prime}=0.1$, and $N_{0}=0.05$.

$$
N(i)=\left(N_{0}+\frac{1+I}{2 \mu} \dot{v}\right)\left(\frac{1+\mu}{1-\mu}\right)^{i}-\frac{1+I}{2 \mu} \dot{v},
$$

so that the effective mass is equal to

$$
m_{1}^{*}=\frac{1+I}{2 \mu}\left[\left(\frac{1+\mu}{1-\mu}\right)^{L}-1\right] .
$$

Thus, the effective inertia in this regime can be much greater than the real mass $L$ of the system. It increases rapidly with $\mu$ and for small values of $\mu$, is at least $1+I$ times the real mass of the array.

From Eq. (33) we get also the following expression for the effective friction force:

$$
F^{*}=\left[\left(\frac{1+\mu}{1-\mu}\right)^{L}-1\right] N_{0} .
$$

In Sec. III, we introduced a global coefficient of friction $M$, which is the "total" friction force $F$ ' on the plane divided by the real mass $L$ of the system. Since a global description implies analytically an effective inertia to be introduced, a description in terms of "effective" quantities is more consistent. Hence, we introduce the effective renormalized coefficient of friction by

$$
M^{*}=\frac{F^{*}}{m^{*}} .
$$

We recall that the acceleration of gravity has been set to 1. From Eqs. (34) and (35), the expression of $M^{*}$ in the regime of collective rolling is given by

$$
M_{1}^{*}=\frac{2 \mu}{1+I} N_{0} .
$$

This is a simple expression in that it depends neither on the applied force nor on the number of particles. In this respect, $M_{1}^{*}$ shows exactly all the features of a Coulombian coefficient of friction.

The confining force $N_{0}$ in this regime is a control parameter of $M_{1}^{*}$. When $N_{0}=0$, the effective coefficient of friction vanishes! The system is hence reduced to a block with no friction on the plane and an effective mass 
greater than its real mass. This has interesting physical implications. For instance, since the effective inertia is greater than the real mass, the speed of sound through the system, due to a small elasticity at contact points, should be less by at least a factor of $\sqrt{1+I}$ than when rotations are absent. On the other hand, since the friction force for small values of $\mu$ is proportional to the number of particles $L$, a small change in the confining force is amplified by a factor $L$ to give a much larger friction force.

(2) When the applied force is greater than $N^{\prime}$, the effective mass begins to increase with the applied force. At the same time, the number of particles in phase 1 decreases and there are more and more particles in phases 2 and 3 . As long as there are particles in phase 1 , the effective mass continues to increase. From Eqs. (12) and (23), it can be shown that the effective mass and coefficient of friction in phase 2 are given by

$$
\begin{aligned}
& m_{2}^{*}=\frac{1}{1-\mu \mu^{\prime}} L \\
& M_{2}^{*}=\mu^{\prime}
\end{aligned}
$$

where $L$ is the number of particles in phase 2. In the same way, for phase 3, from Eqs. (12) and (27), we obtain

$$
\begin{aligned}
m_{3}^{*}= & L, \\
M_{3}^{*}= & \mu^{\prime}-\frac{\mu^{\prime 2}}{L}\left[\left(\frac{1+\mu^{\prime}}{1-\mu^{\prime}}\right)^{L}-1\right] \\
& \times\left(-\mu N(k=0)+\frac{\mu^{\prime}}{2}+\frac{I \omega}{2 \mu^{\prime}}\right) .
\end{aligned}
$$

where $L$ is the length of phase 3 . Here we have taken $\dot{\omega}$ to be an independent parameter which is related essentially on the particle-block coefficient of friction. The effective coefficient of friction in phase 3 is approximately equal to $\mu^{\prime}$. The deviation from this value is of the order of $\mu^{\prime 3}$ when the angular acceleration is zero, and of the order of $\mu^{\prime 2}$ otherwise.

The effective inertia and coefficient of friction of the array can be calculated from those of the phases:

$$
\begin{aligned}
m^{*} & =m_{1}^{*}+m_{2}^{*}+m_{3}^{*}, \\
M^{*} & =\frac{M_{1}^{*} m_{1}^{*}+M_{2}^{*} m_{2}^{*}+M_{3}^{*} m_{3}^{*}}{m_{1}^{*}+m_{2}^{*}+m_{3}^{*}} .
\end{aligned}
$$

As the number of particles in phase 1 decreases, the effective coefficient of friction increases and, at the same time, the effective inertia of the array tends to its real mass.

(3) For a certain value $N^{\prime \prime}$ of the applied force, there will be no particle in phase 1 and the relation between acceleration and applied force becomes linear again. In this regime, the effective mass is practically equal to the real mass of the system, except for small oscillations coming from phase 3. The effective coefficient of friction in this regime is equal to the particle-plane coefficient of friction. Figures 13 and 14 display the variation of effective coefficient of friction and effective inertia as a function of the applied force for an array of 20 particles. $N_{0}$ and $\mu$ are the control parameters of the first regime.

It can be seen from Fig. 13 that the necessary condition for observing the first regime for any number of particles is given by a simple inequality:

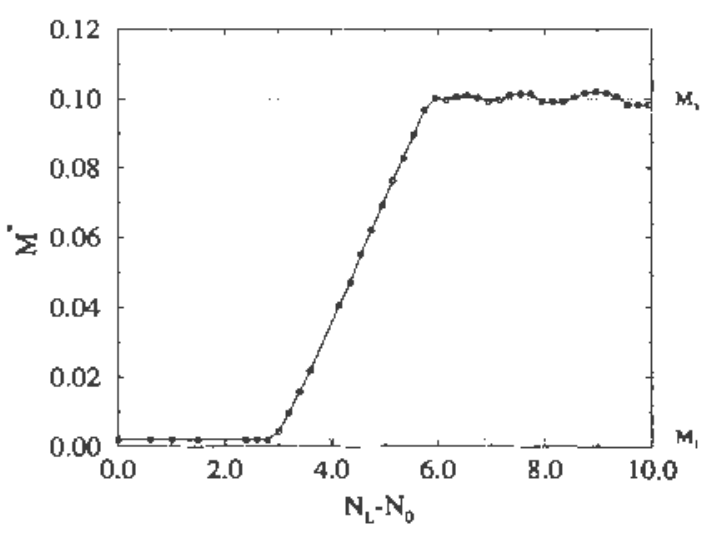

FIG. 13. Variation of the effective coefficient of friction as a function of the applied force for the system of Fig- 12. $M_{1}=2 \mu /(1+I) N_{0}$ is the effective coefficient of friction in phase 1. $M_{3}=\mu^{\prime}$ is the mean value of the coefficient of friction in phases 2 and 3 .

$$
N_{0}<\frac{1+I}{2} \frac{\mu^{\prime}}{\mu} .
$$

When the confining force is small, the system is always in the first regime for small enough values of the applied force. Collective rolling of particles should occur for example at the free surface of sandpiles. Therefore, physical effects due to this mode should be ohservable in real sandpiles.

\section{CONCLUSION}

We have introduced a simple one-dimensional model of particles where the interplay of the dynamics and the contact law (Coulomb's law of friction) leads to a well defined organization of the rotatjons of particles in the steady state. This self-organization has been studied numerically and analyzed in terms of a juxtaposition of "pure modes." The latter have been fully characterized analytically and their respective lengths have been computed. The agreement between the numerical simulations and the theoretical analysis is perfect.

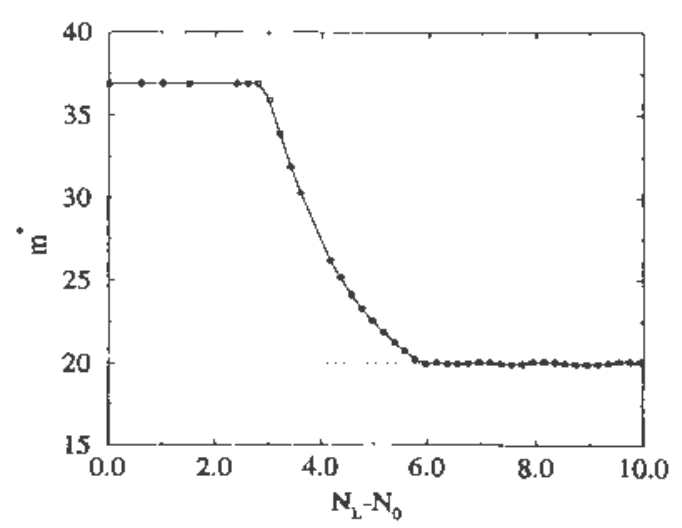

FIG. 14. Variation of the effective mass as a function or the applied force for the system of Fig. 12. 
The translational motion of the array on the plane in the steady state can be described in terms of a global friction force, increasing with the external driving force. However, direct analysis shows that an effective inertia has to be introduced. This in turn requires an "effective" coefficient of friction to be defined, which is different from the "global" coefficient of friction in many respects.

Finally, let us stress the importance of length scales intermediate between the particle size and the system size appearing in rotation modes and force patterns, which may play a significant role in a continumm description of granular media. While this effect is limited here to a one-dimensional geometry, we expect similar qualitative effects in higher dimensionalities. A two-dimensional case is presently under study.

\section{ACKNOWLEDGMENTS}

It is a pleasure to acknowledge useful discussions with D. Bideau, H. J. Herrmann, J. P. Troadec, and D. Wolf. This work was supported by the Groupement de Recherche "Physique des Milieux Hétérogènes Complexes" of the CNRS.
[1] W. Nowacki, in Theory of Micro-polar Elasticity (Springer, Udioe, 1972).

[2] J.-P. Bardet aod J. Proubet, Géotechnique 41, 599 (1991).

[3] Chang and M. Lun, J. Eng. Mech. Div. ASCE 116, 2310 (1990).

[4] P. Evesque and D. Sornette, J. Mech. Behaviors Mater. 5, 261 (1994).

[5] I. M. Schwartz, D. L. Johnson, and S. Feng, Phys. Rev.
Lett. 52,831 (1984).

[6] H. M. Jaeger aod S. Nagel, Science 255, 1523 (1992).

[7] M. Jean, Mechanics of Geometrical Interfaces (Elsevier Science Publishers, New York, 1994).

[8] M. Jean and J. J. Moreau, in Contact Mechanics, edited by A. Curnier, Proceediugs of the Contact Mechanics International Symposium, edited by A. Curnier (Presses Université Romandes, Lausanne 1992), p. 31-48. 